\title{
Genetic Analysis of Consanguineous Pakistani Families with Congenital Stationary Night Blindness
}

\author{
Hafiz Muhammad Azhar Baig a,b,c Muhammad Ansar b,c Afia lqbal ${ }^{d}$ \\ Muhammad Asif Naeem $^{\mathrm{e}}$ Mathieu Quinodoz ${ }^{\mathrm{b}, \mathrm{c}, \mathrm{f}} \quad$ Giacomo Calzetti $^{\mathrm{b}, \mathrm{c}}$ \\ Muhammad lqbal ${ }^{a}$ Carlo Rivoltab,,$f$
}

aDepartment of Biotechnology, Institute of Biochemistry, Biotechnology and Bioinformatics, The Islamia University of Bahawalpur, Bahawalpur, Pakistan; 'Institute of Molecular and Clinical Ophthalmology Basel (IOB), Basel, Switzerland; 'Department of Ophthalmology, University of Basel, Basel, Switzerland; 'Department of Zoology, Lahore College for Women University, Lahore, Pakistan; ${ }^{e}$ Centre of Excellence in Molecular Biology, University of the Punjab, Lahore, Pakistan; fDepartment of Genetics and Genome Biology, University of Leicester, Leicester, UK

\author{
Keywords \\ Congenital stationary night blindness $\cdot R D H 5 \cdot N Y X \cdot S A G$. \\ Consanguinity
}

\begin{abstract}
Introduction: Congenital stationary night blindness (CSNB) is a rare, largely nonprogressive, inherited retinal disorder that can be clinically classified on the basis of fundus and electroretinogram abnormalities. Methods: We analyzed four large consanguineous families from the Southern Punjab region of Pakistan including multiple individuals affected with CSNB. Exome sequencing was performed in probands of all four families; Sanger sequencing was performed in additional members to test co-segregation of the variants identified. Results: We identified two novel and likely pathogenic variants in two pedigrees, namely, NM_002905.4:c.668A>C (p.Gln223Pro) in RDH5 and NM_022567.2:c.908del (p.Gly303ValfsTer45) in NYX. In the two other families, the variants NM_002905.4:c.319G >C (p.Gly107Arg) in RDH5 and NM_000541.5:c.874C>T (p.Arg292Ter) in SAG were identi-
\end{abstract}

karger@karger.com www.karger.com/ore

Karger $\stackrel{\text { ' }}{5}$

BOPEN ACCESS
(C) 2021 The Author(s)

Published by S. Karger AG, Basel

This article is licensed under the Creative Commons Attribution 4.0 International License (CC BY) (http://www.karger.com/Services/ OpenAccessLicense). Usage, derivative works and distribution are permitted provided that proper credit is given to the author and the original publisher. fied. These latter mutations have been reported previously, but not in the Pakistani population. Conclusions: Our findings expand the mutational spectrum of CSNB, in particular within the population of Southern Punjab.

(c) 2021 The Author(s).

Published by S. Karger AG, Basel

\section{Introduction}

Congenital stationary night blindness (CSNB) is a group of heterogenous, largely nonprogressive, inherited retinal disorders that affect bipolar cells, photoreceptor cells, or retinal pigment epithelium cells $[1,2]$. Affected individuals often complain of night vision problems or delayed dark adaptation, but some forms are also associated with a decrease in visual acuity, myopia, strabismus, and nystagmus [3]. Based upon fundus appearance, CSNB can be classified into forms with or without fundus abnormalities, with the former including Oguchi disease and fundus albipunctatus (FAP). The characteristic fea-
Correspondence to:

Muhammad Iqbal, iqbal.khawaja@iub.edu.pk

Carlo Rivolta, carlo.rivolta@iob.ch 
ture of Oguchi disease is the presence of a golden-greyish sheen (most prominent in the peripheral retina) when the fundus is exposed to light, while there is no remarkable fundus alteration upon prolonged dark adaptation [2]. The disappearance of the golden-greyish sheen upon prolonged dark adaptation, known as the Mizuo-Nakamura phenomenon, is considered a pathognomonic clinical feature of Oguchi disease [4]. Conversely, FAP is characterized by small white spots in the retina, which are presumed to contain precursors of 11-cis-retinal. CSNB with largely normal fundus appearance can be divided into complete or incomplete Schubert-Bornschein-type and Riggs-type, based on distinct ERG findings [5-7].

CSNB may follow an autosomal dominant, autosomal recessive, or X-linked pattern of inheritance, due to mutations in genes having a role in phototransduction or subsequent signal transmission to ganglion cells. More specifically, there are at least 19 genes known to be linked to this condition, including CABP4, CACNA1F, CACNA2D4, GNAT1, GPR179, GRK1, GRM6, LRIT3, NYX, PDE6B, RDH5, RHO, RLBP1, RPE65, SAG, SLC24A1, TRPM1, GNB3, and RIMS2 [1,8-10]. Recently, mutations in GUCY2D have also been associated with congenital night blindness with variable progression to mild retinitis pigmentosa (RP) [11]. Finally, a dual phenotype of congenital night blindness and cone-rod dystrophy was described in two Japanese siblings with coexistence of GNAT1 and ABCA4 mutations [12]. Oguchi disease is caused by GRK1 or SAG mutations, and follows an autosomal recessive pattern of inheritance, whereas FAP is mostly associated with recessive mutations in the $\mathrm{RDH} 5$ gene [13-15]. CSNB without fundus abnormalities has greater genetic heterogeneity.

Consanguineous populations represent a powerful tool to study autosomal recessive Mendelian disorders, and the Pakistani population is highly consanguineous, with $>50 \%$ of marriages occurring between cousins, reaching the value of $80 \%$ in some areas $[16,17]$. The Southern region of the Punjab Province is one of such areas. Moreover, nearly half of the population is illiterate [18] and essentially unaware of the risks associated with consanguinity.

Many genes and mutations associated with different genetic disorders have been identified in Pakistani pedigrees. However, the majority of these families originated from Central Punjab and the Sindh Province [19, 20]. Here we studied four consanguineous families with CSNB phenotype from South Punjab and identified novel and likely pathogenic variants in known CSNB genes.

\section{Materials and Methods}

\section{Enrollment of Families}

All families were identified from the Southern region of the Punjab Province in Pakistan and were enrolled on the basis of the number of affected individuals (at least two affected individuals), presence/absence of consanguinity (only consanguineous pedigrees), and pattern of inheritance (only recessive or X-linked, by pedigree analysis). More specifically, the families described here are part of a larger cohort of patients, all displaying inherited retinal dystrophies, but the four pedigrees analyzed are the only ones with clear-cut CSNB phenotypes. A detailed questionnaire was designed to rule out the presence of a syndromic form of inherited retinal disorders. Written consent was obtained from all the participants of the study or from their parents/legal guardians. This study was approved by the Bioethics Committees of the Islamia University of Bahawalpur, Pakistan, and the Ethikkommission Nordwest- und Zentralschweiz.

\section{Clinical Examination}

Clinical examination of three families, namely, PKIURP03, PKIURP06, and PKIURP07, was performed by an ophthalmologist. This examination included the collection of medical history and funduscopy (shown in Fig. 1). Clinical examination of affected individuals from family PKIURP51 was not performed, as they live in a village where the access was difficult for ophthalmologists. In this family, the assessment of the disease was performed by the evaluation of the available medical history and by their descriptive phenotype.

\section{Collection of Blood Samples and DNA Extraction}

Ten milliliters of intravenous blood were collected from selected members of the enrolled families, in tubes containing EDTA as an anticoagulant. DNA was extracted by using standard techniques and quantified by spectrophotometry. DNA quality was assessed by gel electrophoresis, on $1 \%$ agarose gels.

\section{Exome Sequencing and Segregation Analysis}

Exome sequencing (ES) was performed in probands from all four families by using the Twist Comprehensive Exome Panel (Twist bioscience) and sequenced on a HiSeq 4000 instrument (Illumina) with an average coverage of 100-120X at each nucleotide position. Novoalign software (V3.08.00; Novocraft Technologies, Selangor, Malaysia) was used for mapping raw reads to the human genome reference sequence (build hg19). HaplotypeCaller (GATK, v.4.0.3.0) was used for Base Quality score recalibration. Picard (v. 2.14.0-SNAPSHOT) was used for the removal of duplicates. Small InDels and SNVs were detected by using GATK (GATK v4.0) [21]. Homozygosity mapping was performed by the use of Automap [22]. Allele frequencies were checked by the use of the gnomAD database (https://gnomad.broadinstitute.org/), and conservation of variants was performed by using GERP++ [23]. ANNOVAR [24] and in-house scripts [25] were used for the assessment of the pathogenicity of genetic variants.

\section{Results}

\section{Clinical Findings}

Thirteen patients from 4 unrelated families were studied. Family PKIURP03 included three patients. Individu- 
Fig. 1. Fundus images of proband II:1 from family PKIURP03 (a), proband V:3 from family PKIURP06 (b), and proband II:2 from family PKIURP07 (c). Yellow arrows indicate retinal white spots of FAP, while cyan arrows indicate the central macular area, relatively spared from the presence of white spots. Green arrows indicate areas of greyish discoloration of the fundus in Oguchi disease. FAP, fundus albipunctatus.

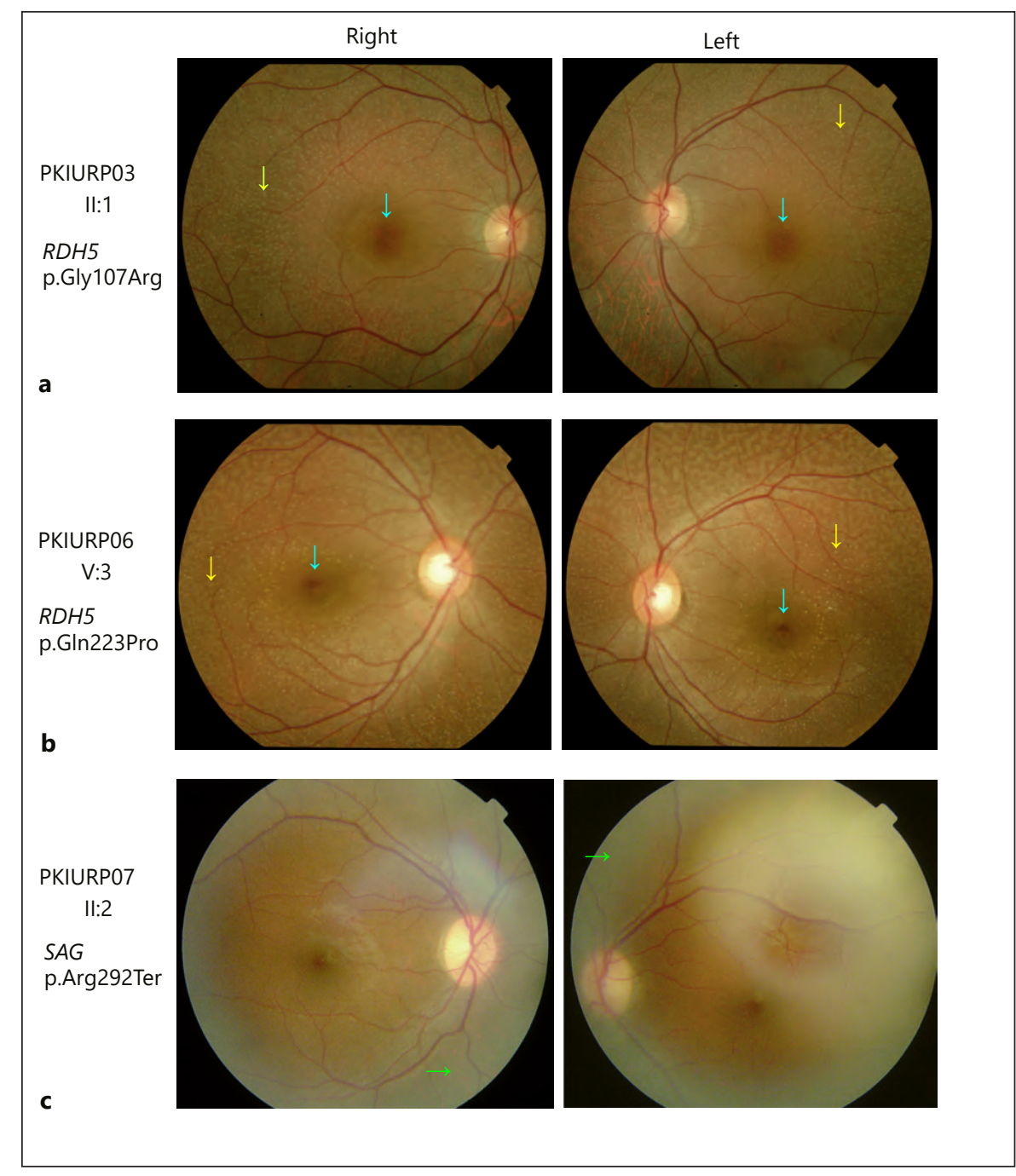

al II: 1 was a male of 29 years, whereas II:2 was a female of 17 years, and individual II:3 was a female of 13 years. All three patients had night vision problems since early ages. Fundus images of the proband (II:1) showed the presence of scattered white spots in the retinal mid-periphery with relative macular sparing, a characteristic of FAP. A localized, symmetrical area of greater choroidal visibility in the nasal inferior fundus was noted in both eyes, but optic disks and retinal vessels were of normal appearance (shown in Fig. 1a).

Family PKIURP06 included four affected individuals in two loops. All affected individuals were of 12 years or younger, and their parents became aware of their nyctalopia when they were toddlers. Fundus photograph of one proband (V:3) showed the presence of scattered whitish spots in the retinal mid-periphery and, to a lesser extent, in the macula of both eyes (shown in Fig. 1b). Based on these findings, the affected probands of families PKIURP03 and PKIURP06 were given a diagnosis of FAP.

Family PKIURP07 included two patients. Individual II: 1 was a female aged 18 years, whereas individual II:2 was a male aged 15 years, both reporting seemingly nonprogressive nyctalopia since early ages. Clinical history could not be further expanded since both parents were deceased. Fundus images of the proband (II:2), taken under light-adapted conditions, showed areas of greyish discoloration beyond the vascular arcades of both eyes without signs of RP, such as bone-spicule pigmentation, optic disc pallor, or vascular attenuation (shown in Fig. 1c), which is compatible with Oguchi disease.

Family PKIURP51 included four affected brothers and three unaffected sisters. The eldest patient (II:1) was 30 years old at the time of analysis. Two affected individuals were available at the time of sampling, II.1 and II.2 (28 


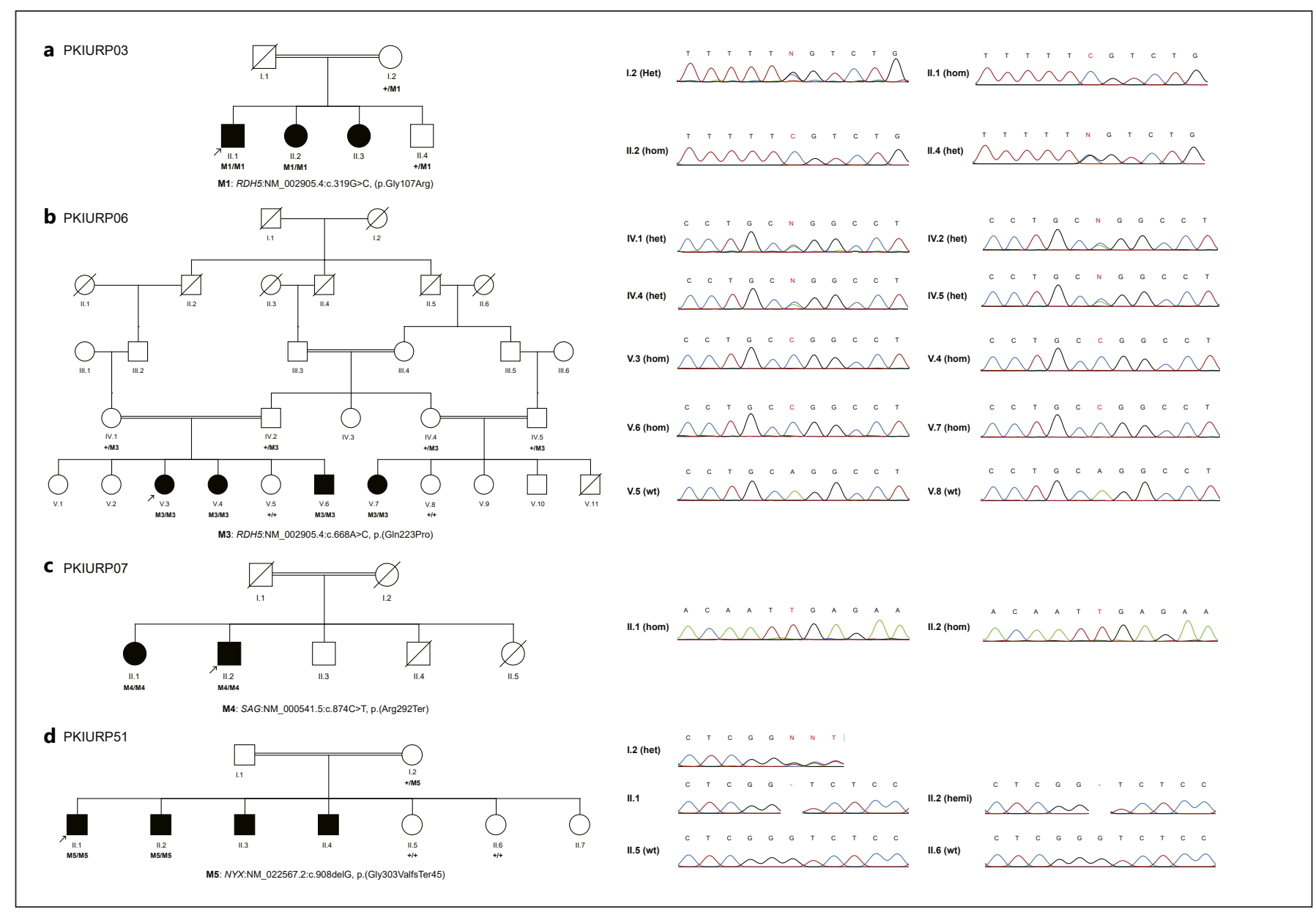

Fig. 2. a-d Segregation analysis of the variants identified within the pedigrees analyzed. Probands from every family are indicated by the arrow sign. In total, there were 13 patients (black squares and circles); however, only 10 were genetically analyzed (mutations are reported below each patient's symbol). In the electropherograms, the red nucleotide corresponds to the variant, "N" to heterozygous calls, and "-" to deleted nucleotides. In subject I.2 of family PKIURP51, the hetrozygous deletion of one base pair results in the shifting of the sequence; therefore, there are multiple "N" calls. het, heterozygote; hom, homozygote; hemi, hemizygote; wt, wild type.

years old), both reporting nonprogressive difficulties in seeing at night associated with high myopia. No additional clinical data were available.

\section{Molecular Findings}

Ten patients were genetically analyzed (Fig. 2). ES analysis of probands from all four families (PKIURP03, PKIURP06, PKIURP07, and PKIURP51) revealed the presence of four variants in three different genes already linked to CNSB.

In family PKIURP03, we identified the homozygous variant NM_002905.4:c.319G>C (p.Gly107Arg) in the $R D H 5$ gene, which was absent from the gnomAD data- base. This variant segregated with the condition within the family (shown in Fig. 2). The effect of this variant on the three-dimensional structure and on the function of the retinol dehydrogenase enzyme (encoded by $\mathrm{RDH} 5$ gene) was predicted by HOPE [26] to be likely pathogenic because of the larger size and the positive charge of arginine with respect to glycine.

Family PKIURP06 carried a novel homozygous variant, NM_002905.4:c.668A >C (p.Gln223Pro) in the RDH5 gene. This variant, again, co-segregated with disease in a Mendelian recessive fashion in 10 individuals (4 affected and 6 unaffected individuals, shown in Fig. 2). This missense variant was not present in gnomAD, and HOPE 
predicted pathogenicity based on the larger size of glutamine with respect to proline and the presence of this amino acid residue within the alpha helical region of retinol dehydrogenase.

The proband of family PKIURP07 with Oguchi disease showed an already-reported pathogenic homozygous variant in the SAG gene NM_000541.5:c.874C>T (p.Arg292Ter) [27-30]. This mutation is a stopgain and has an allele frequency 0.000359 in gnomAD South Asia; the variant segregated with disease in both patients from this pedigree (shown in Fig. 2).

Pedigree analysis of family PKIURP51 suggested an Xlinked pattern of inheritance for CSNB. Exome data of the proband identified a deletion in the NYX gene NM_022567.2:c.908del (p.Gly303ValfsTer45), on chromosome $\mathrm{X}$, which was also absent from gnomAD. Segregation analysis of this novel deletion in the family confirmed indeed an X-associated inheritance of the mutation (shown in Fig. 2).

\section{Discussion}

In this study, we analyzed four Pakistani families with CSNB by performing ES and segregation studies. Two families (PKIURP03 and PKIURP06) had FAP, one (PKIURP07) had Oguchi disease, and the fourth family (PKIURP51) had NYX-associated CSNB.

Families PKIURP03 and PKIURP06, with the phenotype of FAP, were found to carry the homozygous variants p.Gly107Arg and p.Gln223Pro in $R D H 5$, respectively. $\mathrm{RDH} 5$ encodes retinol dehydrogenase 5 , an essential enzyme for the visual cycle [31]. This enzyme causes the catalytic conversion of 11-cis-retinol to 11-cis-retinal chromophore in the retinal pigment epithelium [32]. The variant p.Gly107Arg identified in family PKIURP03 has already been reported in 2 compound heterozygous [ 33 , $34]$ and 3 homozygous [35, 36] Japanese patients with FAP and retinal degenerative changes, respectively. Interestingly, one homozygous patient showed sectorial retinochoroidal degenerative changes in the inferonasal quadrant resonating with the fundus findings in our proband [36]. The p.Gln223Pro variant identified in the second $\mathrm{RDH} 5$ family is instead a novel variant.

Family PKIURP07 was found to carry a homozygous variant (p.Arg292Ter) in the $S A G$ gene. This gene encodes the arrestin 1 protein $[37,38]$. It is specifically expressed in rods and has a regulatory function within the phototransduction cascade. Mutations in $S A G$ have been reported in association with Oguchi disease as well as au- tosomal recessive RP [39]. The homozygous variant $\mathrm{p}$. Arg292Ter has been previously identified only in association with Oguchi disease $[27,29]$, which is in line with the findings in our proband.

Male patients of family PKIURP51 carried the hemizygous variant p.Gly303ValfsTer45 in NYX. This frameshift deletion is located in the second and last exon of the gene, therefore likely leading to an aberrant mRNA possibly escaping nonsense-mediated mRNA decay. The NYX gene encodes nyctalopin, a 481-amino acid protein involved in the localization of the TRPM1 protein in depolarizing bipolar cells [40]. Mutations in NYX result in the indirect mislocalization of TRPM1, which in turn causes X-linked CSNB [40-42]. Affected individuals from family PKIURP51 had night blindness with high myopia, similar to the phenotype described by Yip et al. [43]. Many reports suggest that mutations in the NYX gene are a common cause of X-linked CSNB in the Chinese and in other populations [43-46]. However, to the best of our knowledge, this is the first report of a NYX mutation in a Pakistani pedigree.

One important limitation of this study is the clinical characterization of the patients, including the lack of electrophysiological assessment and other analyses of visual function or retinal structure, such as optical coherence tomography and fundus autofluorescence [47]. Furthermore, we could not document the Mizuo-Nakamura phenomenon in the proband of family PKIURP07, and we based the clinical diagnosis only on medical history and questionnaire in the patients from family PKIURP51. The nonprogressive nature of night blindness supported a clinical diagnosis of CSNB rather than other progressive phenotypes such as RP. In families PKIURP03, 06, and 07 fundus photographs were consistent with phenotypes of FAP or Oguchi disease, showing no typical signs of RP such as bone spicules, vascular attenuation, or optic disk waxy pallor. Finally, our molecular findings correlated with the available clinical phenotype in all the examined probands. In conclusion, following the molecular analysis of four Pakistani families with CSNB, we identified two novel and likely pathogenic variants in the $\mathrm{RDH} 5$ and NYX genes, broadening the mutational spectrum of this rare retinal condition.

\section{Acknowledgments}

We are very much thankful to all individuals who participated in the current study. We would also like to thank Marc Folcher, Ji Hoon Han, and Mukhtar Ullah for helping in preparing the figures and for reviewing the manuscript. 


\section{Statement of Ethics}

This study protocol was reviewed and approved by the Institutional Bioethics Committee (IBC), the Islamia University of Bahawalpur, Pakistan, approval number 3837/ORIC, and the Ethikkommission Nordwest-und Zentralschweiz, Switzerland, approval number 2019-01660. Consent to participate statement: Written informed consent was obtained from all participants or their parents/legal guardians.

\section{Conflict of Interest Statement}

The authors declare no conflict of interests.

\section{Funding Sources}

This research received funding from the Swiss National Science Foundation (Grant \#176097 to CR). G.C. was supported by a Diana Davis Spencer Clinical Research Fellowship Award from the Foundation Fighting Blindness.

\section{Author Contributions}

H.M.A.B., M.A., G.C., and C.R. wrote the manuscripts. A.I., H.M.A.B., and M.A. analyzed exome data and performed Sanger sequencing. M.A.N. and M.I. examined the patients. M.Q. contributed in the data analysis. M.I. and C.R. supervised the study.

\section{Data Availability Statement}

Data generated or analyzed during this study are included in this article. ES data have purposefully not been made available in full, in order to comply with current guidelines on the protection of personal information leading to possible identification. Further inquiries can be directed to the corresponding authors.

\section{References}

1 Zeitz C, Robson AG, Audo I. Congenital stationary night blindness: an analysis and update of genotype-phenotype correlations and pathogenic mechanisms. Prog Retin Eye Res. 2015 Mar;45:58-110.

2 Baldwin AN, Robson AG, Moore AT, Duncan JL. Ryan's retina. 2018.

3 Bijveld MM, Florijn RJ, Bergen AA, van den Born LI, Kamermans M, Prick L, et al. Genotype and phenotype of 101 dutch patients with congenital stationary night blindness. Ophthalmology. 2013 Oct;120(10):2072-81.

4 Mizuo G. A new discovery in dark adaptation in Oguchi disease. Acta Soc Ophthal Jpn. 1913;17:1148-50.

5 Schubert G, Bornschein H. [Analysis of the human electroretinogram]. Ophthalmologica. 1952 Jun;123(6):396.

6 Riggs LA. Electroretinography in cases of night blindness. Am J Ophthalmol. 1954 Jul; 38(12):70-8.

7 Miyake Y, Yagasaki K, Horiguchi M, Kawase $\mathrm{Y}$, Kanda T. Congenital stationary night blindness with negative electroretinogram. A new classification. Arch Ophthalmol. 1986 Jul;104(7):1013-20.

8 Vincent A, Audo I, Tavares E, Maynes JT, Tumber A, Wright T, et al. Biallelic mutations in GNB3 cause a unique form of autosomalrecessive congenital stationary night blindness. Am J Hum Genet. 2016 May 5;98(5):1011-9.

9 Almutairi F, Almeshari N, Ahmad K, Magliyah MS, Schatz P. Congenital stationary night blindness: an update and review of the disease spectrum in Saudi Arabia. Acta Ophthalmol. 2021 Sept;99(6):581-91.
10 Mechaussier S, Almoallem B, Zeitz C, Van Schil K, Jeddawi L, Van Dorpe J, et al. Loss of function of RIMS2 causes a syndromic congenital cone-rod synaptic disease with neurodevelopmental and pancreatic involvement. Am J Hum Genet. 2020 Sep 3;107(3):580.

11 Stunkel ML, Brodie SE, Cideciyan AV, Pfeifer WL, Kennedy EL, Stone EM, et al. Expanded retinal disease spectrum associated with autosomal recessive mutations in GUCY2D. Am J Ophthalmol. 2018 Jun;190:58-68.

12 Hayashi T, Hosono K, Kurata K, Katagiri S, Mizobuchi K, Ueno S, et al. Coexistence of GNAT1 and ABCA4 variants associated with Nougaret-type congenital stationary night blindness and childhood-onset cone-rod dystrophy. Doc Ophthalmol. 2020 Apr;140(2): $147-57$.

13 Fuchs S, Nakazawa M, Maw M, Tamai M, Oguchi Y, Gal A. A homozygous 1-base pair deletion in the arrestin gene is a frequent cause of Oguchi disease in Japanese. Nat Genet. $1995 \mathrm{Jul} ; 10(3): 360-2$.

14 Yamamoto S, Sippel KC, Berson EL, Dryja TP. Defects in the rhodopsin kinase gene in the Oguchi form of stationary night blindness. Nat Genet. $1997 \mathrm{Feb} ; 15(2): 175-8$.

15 Yamamoto H, Simon A, Eriksson U, Harris E, Berson EL, Dryja TP. Mutations in the gene encoding 11-cis retinol dehydrogenase cause delayed dark adaptation and fundus albipunctatus. Nat Genet. 1999 Jun;22(2):188-91.

16 National Institute of Population Studies. Pakistan demographic and health Survey; Pakistan. 2013. Maryland, MD, USA: NIPS; 2013.
17 Riaz M, Tiller J, Ajmal M, Azam M, Qamar R, Lacaze P. Implementation of public health genomics in Pakistan. Eur J Hum Genet. 2019 Oct;27(10):1485-92.

18 Abdul R, Luan J, Imran H. The province-wise literacy rate in Pakistan and its impact on the economy. Pac Sci Rev B Humanit Soc Sci. 2015;1(3):140-4

19 Saqib MA, Nikopoulos K, Ullah E, Sher Khan F, Iqbal J, Bibi R, et al. Homozygosity mapping reveals novel and known mutations in Pakistani families with inherited retinal dystrophies. Sci Rep. 2015 May 6;5:9965.

20 Chen J, Wang Q, Cabrera PE, Zhong Z, Sun $\mathrm{W}$, Jiao X, et al. Molecular genetic analysis of pakistani families with autosomal recessive congenital cataracts by homozygosity screening. Invest Ophthalmol Vis Sci. 2017 Apr 1; 58(4):2207-17.

21 DePristo MA, Banks E, Poplin R, Garimella KV, Maguire JR, Hartl C, et al. A framework for variation discovery and genotyping using next-generation DNA sequencing data. Nat Genet. 2011 May;43(5):491-8.

22 Quinodoz M, Peter VG, Bedoni N, Royer Bertrand B, Cisarova K, Salmaninejad A, et al. AutoMap is a high performance homozygosity mapping tool using next-generation sequencing data. Nat Commun. 2021 Jan 22; 12(1):518.

23 Davydov EV, Goode DL, Sirota M, Cooper GM, Sidow A, Batzoglou S. Identifying a high fraction of the human genome to be under selective constraint using GERP++. PLoS Comput Biol. 2010 Dec 2;6(12):e1001025. 
24 Wang K, Li M, Hakonarson H. ANNOVAR: functional annotation of genetic variants from high-throughput sequencing data. $\mathrm{Nu}-$ cleic Acids Res. 2010 Sep;38(16):e164.

25 Peter VG, Nikopoulos K, Quinodoz M, Granse L, Farinelli P, Superti-Furga A, et al. A novel missense variant in IDH3A causes autosomal recessive retinitis pigmentosa. Ophthalmic Genet. 2019 Apr;40(2):177-81.

26 Venselaar H, Te Beek TA, Kuipers RK, Hekkelman ML, Vriend G. Protein structure analysis of mutations causing inheritable diseases. An e-Science approach with life scientist friendly interfaces. BMC Bioinformatics. 2010 Nov 8;11:548.

27 Nakamura M, Yamamoto S, Okada M, Ito S, Tano Y, Miyake Y. Novel mutations in the arrestin gene and associated clinical features in Japanese patients with Oguchi's disease. Ophthalmology. 2004 Jul;111(7):1410-4.

28 O'Sullivan J, Mullaney BG, Bhaskar SS, Dickerson JE, Hall G, O'Grady A, et al. A paradigm shift in the delivery of services for diagnosis of inherited retinal disease. J Med Genet. 2012 May;49(5):322-6.

29 Wang X, Wang H, Sun V, Tuan HF, Keser V, Wang K, et al. Comprehensive molecular diagnosis of 179 Leber congenital amaurosis and juvenile retinitis pigmentosa patients by targeted next generation sequencing. J Med Genet. 2013 Oct;50(10):674-88

30 Li L, Chen Y, Jiao X, Jin C, Jiang D, Tanwar $\mathrm{M}$, et al. Homozygosity mapping and genetic analysis of autosomal recessive retinal dystrophies in 144 consanguineous Pakistani families. Invest Ophthalmol Vis Sci. 2017 Apr 1; 58(4):2218-38.

31 Simon A, Lagercrantz J, Bajalica-Lagercrantz S, Eriksson U. Primary structure of human 11-cis retinol dehydrogenase and organization and chromosomal localization of the corresponding gene. Genomics. 1996 Sep 15; 36(3):424-30
32 Farjo KM, Moiseyev G, Takahashi Y, Crouch RK, Ma JX. The 11-cis-retinol dehydrogenase activity of RDH10 and its interaction with visual cycle proteins. Invest Ophthalmol Vis Sci. 2009 Nov;50(11):5089-97.

33 Nakamura M, Hotta Y, Tanikawa A, Terasaki $\mathrm{H}$, Miyake Y. A high association with cone dystrophy in fundus albipunctatus caused by mutations of the RDH5 gene. Invest Ophthalmol Vis Sci. 2000 Nov;41(12):3925-32.

34 Katagiri S, Hayashi T, Nakamura M, Mizobuchi K, Gekka T, Komori S, et al. RDH5-related fundus albipunctatus in a large japanese cohort. Invest Ophthalmol Vis Sci. 2020 Mar 9; 61(3):53.

35 Hotta K, Nakamura M, Kondo M, Ito S, Terasaki H, Miyake Y, et al. Macular dystrophy in a Japanese family with fundus albipunctatus. Am J Ophthalmol. 2003 Jun;135(6):917-9.

36 Sato M, Oshika T, Kaji Y, Nose H. A novel homozygous Gly107Arg mutation in the RDH5 gene in a Japanese patient with fundus albipunctatus with sectorial retinitis pigmentosa. Ophthalmic Res. 2004 Jan-Feb;36(1): 43-50.

37 Kohout TA, Lin FS, Perry SJ, Conner DA, Lefkowitz RJ. beta-Arrestin 1 and 2 differentially regulate heptahelical receptor signaling and trafficking. Proc Natl Acad Sci U S A. 2001 Feb 13;98(4):1601-6.

38 Luttrell LM, Lefkowitz RJ. The role of betaarrestins in the termination and transduction of G-protein-coupled receptor signals. J Cell Sci. 2002 Feb 1;115(Pt 3):455-65.

39 Nishiguchi KM, Ikeda Y, Fujita K, Kunikata $\mathrm{H}$, Akiho M, Hashimoto K, et al. Phenotypic features of oguchi disease and retinitis pigmentosa in patients with S-antigen mutations: a long-term follow-up study. Ophthalmology. 2019 Nov;126(11):1557-66.
40 Pearring JN, Bojang P Jr, Shen Y, Koike C, Furukawa T, Nawy S, et al. A role for nyctalopin, a small leucine-rich repeat protein, in localizing the TRP melastatin 1 channel to retinal depolarizing bipolar cell dendrites. J Neurosci. 2011 Jul 6;31(27):10060-6.

41 Bech-Hansen NT, Naylor MJ, Maybaum TA, Sparkes RL, Koop B, Birch DG, et al. Mutations in NYX, encoding the leucine-rich proteoglycan nyctalopin, cause X-linked complete congenital stationary night blindness. Nat Genet. 2000 Nov;26(3):319-23.

42 Khan NW, Kondo M, Hiriyanna KT, Jamison JA, Bush RA, Sieving PA. Primate retinal signaling pathways: suppressing ON-pathway activity in monkey with glutamate analogues mimics human CSNB1-NYX genetic night blindness. J Neurophysiol. 2005 Jan;93(1): 481-92.

43 Yip SP, Li CC, Yiu WC, Hung WH, Lam WW, Lai MC, et al. A novel missense mutation in the NYX gene associated with high myopia. Ophthalmic Physiol Opt. 2013 May;33(3): 346-53.

44 Leroy BP, Budde BS, Wittmer M, De Baere E Berger W, Zeitz C. A common NYX mutation in Flemish patients with X linked CSNB. Br J Ophthalmol. 2009 May;93(5):692-6.

45 Dai S, Ying M, Wang K, Wang L, Han R, Hao $\mathrm{P}$, et al. Two novel NYX gene mutations in the Chinese families with X-linked congenital stationary night blindness. Sci Rep. 2015 Aug 3;5:12679.

46 Ivanova ME, Zolnikova IV, Gorgisheli KV, Atarshchikov DS, Ghosh P, Barh D. Novel frameshift mutation in NYX gene in a Russian family with complete congenital stationary night blindness. Ophthalmic Genet. 2019 Dec;40(6):558-63.

47 Calzetti G, Levy RA, Cideciyan AV, Garafalo AV, Roman AJ, Sumaroka A, et al. Efficacy outcome measures for clinical trials of USH2A caused by the common c.2299delG mutation. Am J Ophthalmol. 2018 Sep;193:114-29. 